\title{
Applying the Retarded Solutions of Electromagnetic Fields to Transmission Line RLGC Modeling
}

\author{
Peng Ye ${ }^{1,2}$, Brandon Gore ${ }^{1,3}$, Paul Huray ${ }^{1}$ \\ ${ }^{1}$ College of Engineering and Computing, University of South Carolina, Columbia, SC, USA \\ ${ }^{2}$ Oracle Corporation, Redwood City, CA, USA \\ ${ }^{3}$ Intel Corporation, Columbia, SC, USA \\ *corresponding author, E-mail: Brandon.Gore@intel.com
}

\begin{abstract}
The RLGC model, and its variations, is one of the most common techniques to simulate transmission lines. The RLGC model uses circuit network elements consisting of Resistance R, Inductance L, Conductance G and Capacitance $\mathrm{C}$ (per unit length) to represent a small segment of the transmission line, and then cascades multiple segments to simulate the transmission line of arbitrary length. Typically, the parameters in RLGC model are extracted from the propagation constant $\gamma$ and characteristic impedance $Z_{o}$ of the transmission line which are found using numerical simulation methods. These resulting RLGC parameters for multi-GHz signaling are usually frequency-dependent. This paper introduces an analytical approach to extract RLGC parameters to simulate a transmission line, which results in a different model, the RLGC(p) model.
\end{abstract}

\section{Introduction}

Maxwell's Equations have been widely studied and used since their publication in 1861 by the Scottish physicist and mathematician James Clerk Maxwell. While Maxwell's Equations may appear in different forms, a common key aspect is that these equations specify the relationship of electric field intensity $(\vec{E})$, magnetic field intensity $(\vec{H})$, electric charge density $(\rho)$ and electric current density $(\vec{J})$ at a particular space-time ${ }^{[2]},\left(\vec{x}^{\prime}, t^{\prime}\right)$. For example:

$$
\begin{aligned}
& \vec{\nabla} \times \vec{E}\left(\vec{x}^{\prime}, t^{\prime}\right)=-\partial \vec{B}\left(\vec{x}^{\prime}, t^{\prime}\right) / \partial t^{\prime} \\
& \vec{\nabla} \times \vec{B}\left(\vec{x}^{\prime}, t^{\prime}\right)=\mu \vec{J}\left(\vec{x}^{\prime}, t^{\prime}\right)+\mu \varepsilon \partial \vec{E}\left(\vec{x}^{\prime}, t^{\prime}\right) / \partial t^{\prime} \\
& \vec{\nabla} \cdot \vec{E}\left(\vec{x}^{\prime}, t^{\prime}\right)=\rho\left(\vec{x}^{\prime}, t^{\prime}\right) / \varepsilon \\
& \vec{\nabla} \cdot \vec{B}\left(\vec{x}^{\prime}, t^{\prime}\right)=0
\end{aligned}
$$

Where $\vec{B}\left(\vec{x}^{\prime}, t^{\prime}\right)=\mu \vec{H}\left(\vec{x}^{\prime}, t^{\prime}\right)$ is the magnetic flux density at point $\vec{x}^{\prime}$, time $t^{\prime}$, and $\mu$ is the permeability of the medium.

To analyze electromagnetic field propagation we need to find the causal relationship between different space-times for the source $\left(\vec{x}^{\prime}, t^{\prime}\right)$ and the observation point $(\vec{x}, t)$. Since electromagnetic fields propagate in the medium with a finite propagation speed, denoted as $v$, there is a time difference in the relationship of electromagnetic fields at the source and the observation point. This phenomenon is known as time retardation and the time difference can be described as $t-$ $t^{\prime}=\left|\vec{x}^{\prime}-\vec{x}\right| / v$.

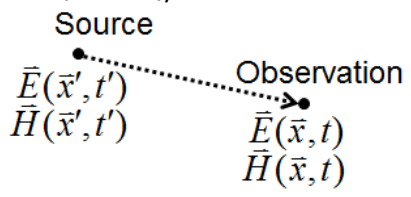

Electrodynamics

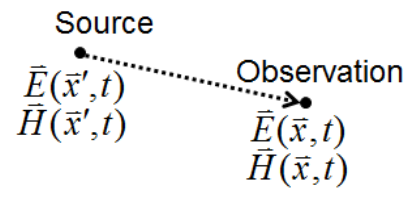

Electrostatic
Figure 1: Time Retardation of Electromagnetic Fields

For Electrostatic fields where the source does not change over time: $\vec{E}\left(\vec{x}^{\prime}, t\right)=\vec{E}\left(\vec{x}^{\prime}, t^{\prime}\right)$ and $\vec{H}\left(\vec{x}^{\prime}, t\right)=\vec{H}\left(\vec{x}^{\prime}, t^{\prime}\right)$; therefore, the electromagnetic fields at the source and the observation point can have the same time component, $t$, which makes the propagation appear instantaneous and renders the time retardation irrelevant. However, for time-varying electromagnetic fields, the time component $t^{\prime}$ and $t$ must be treated differently, and the derivative on $t^{\prime}$ will result in extra terms as $t^{\prime}$ is correlated $t, \vec{x}^{\prime}, \vec{x}$. In other words, a comprehensive solution of electromagnetic fields that addresses time retardation must include these extra terms. We call such a solution the retarded solution of electromagnetic fields. It should be noted that the solution for Electrostatic fields is a special case of the retarded solution where the extra terms are zero due to $t^{\prime}=t$.

The retarded solution of electromagnetic fields can be derived from Maxwell's Equations with a Green's Function. The retarded solution for point source, which is also known as the Jefimenko's Equations ${ }^{[3]}$, can be found as:

$$
\begin{aligned}
& \vec{E}(\vec{x}, t)=\frac{1}{4 \pi \varepsilon}\left[\frac{\vec{r}}{R^{2}}\left[\rho\left(\vec{x}^{\prime}, t^{\prime}\right)\right]_{r e t}+\frac{\vec{r}}{v R} \frac{\left[\partial \rho\left(\vec{x}^{\prime}, t^{\prime}\right)\right]_{r e t}}{\partial t}-\frac{1}{v^{2} R} \frac{\left[\partial \vec{J}\left(\vec{x}^{\prime}, t^{\prime}\right)\right]_{r e t}}{\partial t}\right] \\
& \vec{B}(\vec{x}, t)=\frac{\mu}{4 \pi}\left[\frac{1}{R^{2}}\left[\vec{J}\left(\vec{x}^{\prime}, t^{\prime}\right)\right]_{r e t} \times \vec{r}+\frac{1}{v R} \frac{\left[\partial \vec{J}\left(\vec{x}^{\prime}, t^{\prime}\right)\right]_{r e t}}{\partial t} \times \vec{r}\right]
\end{aligned}
$$

With a Fourier transform, this can be converted to Frequency Domain: 


$$
\begin{aligned}
& \vec{E}(\vec{x}, \omega)=\frac{1}{4 \pi \varepsilon R^{2}}\left[\vec{r}\left(1+j \frac{\omega R}{v}\right) \rho\left(\vec{x}^{\prime}, \omega\right) e^{-j \omega R / v}-j \frac{\omega R}{v^{2}} \vec{J}\left(\vec{x}^{\prime}, \omega\right) e^{-j \omega R / v}\right] \\
& \vec{B}(\vec{x}, \omega)=\frac{\mu}{4 \pi R^{2}}\left(1+j \frac{\omega R}{v}\right)\left[\vec{J}\left(\vec{x}^{\prime}, \omega\right) e^{-j \omega R / v} \times \vec{r}\right]
\end{aligned}
$$

The solution for other types of sources can be developed based on the solution for a point source.

In the above equations, the term $j \frac{\omega R}{v}$ is introduced due to consideration of the retardation, and the ratio $\frac{\omega R}{v}$ can be used as an indicator for the impact of retardation. When the frequency is low, the electrical distance between source and observation point is small, $\frac{\omega R}{v} \ll 1$, and the retarded solution of electromagnetic fields can be approximated by the static solution $^{[1]}$. However, Feynman emphasized that the terms representing time retardation should not be omitted when they become significant ${ }^{[6]}$. Also, for discussion in future sections, it should be recalled that a phase shift in the frequency domain leads to a time retardation in the time domain ${ }^{[2]}$.

\section{Theoretical Case Study}

The retarded solution of electromagnetic fields provides an analytical way to solve the electromagnetic fields. In this section, we use it to solve the test case of a simplified transmission line. For this test case, we use a lossless thin straight line source along the $z$ direction to represent the transmission line and a flat PEC plane at distance $h$ away from the line source to represent the reference plane. We fill the entire space with uniform, lossless medium with $\varepsilon=$ $4 \varepsilon_{0}$ and $\mu=\mu_{0}$ in which the electromagnetic fields propagate at velocity, $v$. As a simplification, the model is extended to infinity to avoid the need of handling any boundary condition.

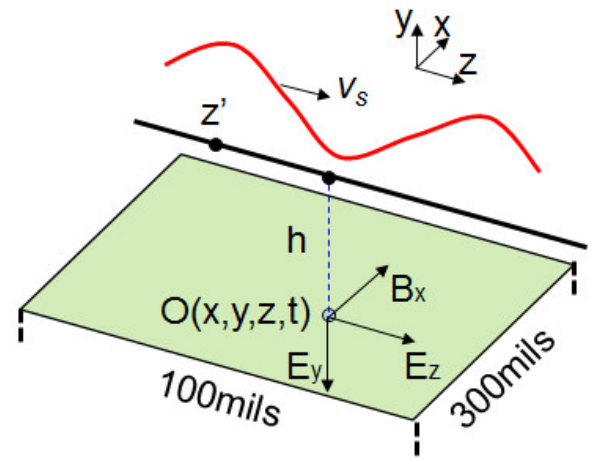

Figure 2: Case Study

For demonstration purposes, a source charge density, $\rho\left(z^{\prime}, t\right)=\alpha_{s}\left(z^{\prime}\right) Q \cos \left(k_{s} z^{\prime}-\omega t-\varphi\right)$, is assumed to be a sinusoidal wave where charge, $Q$, and attenuation, $\alpha_{s}$, are unity and frequency is $100 \mathrm{GHz}$ (refer to Section 3.2 of reference [1]).

We observe the electromagnetic fields on the surface of the PEC reference plane, for $h=10$ mils and $h=100 \mathrm{mils}$, respectively. We plot the magnitude of the fields in color where positive peak is in red and negative peak is in blue.

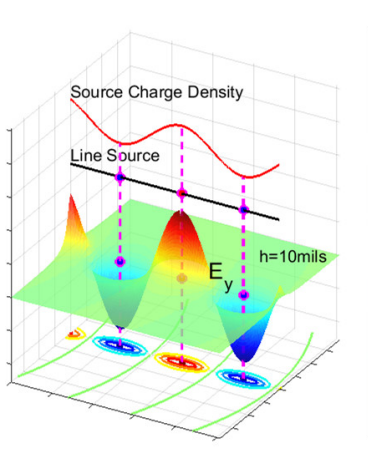

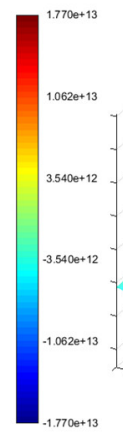

(a) $h=10$ mils

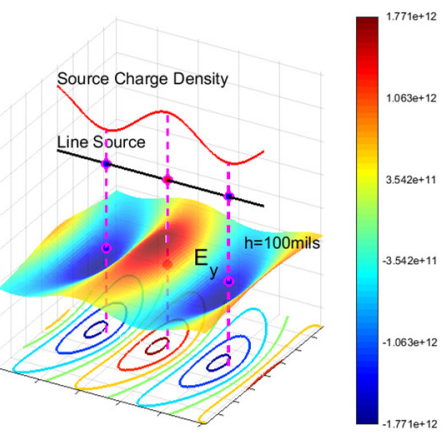

(b) $h=100 \mathrm{mils}$
Figure 3: $\left|\vec{E}_{y}\right|$ magnitude plot, oblique view

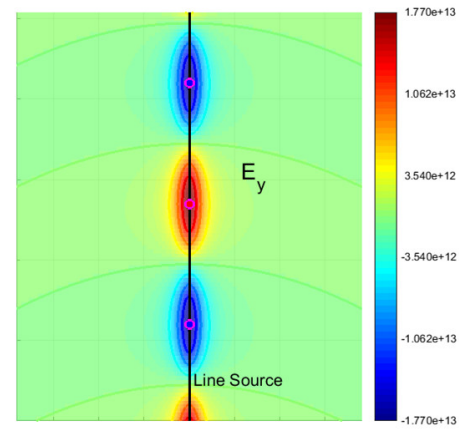

(a) $h=10 \mathrm{mils}$

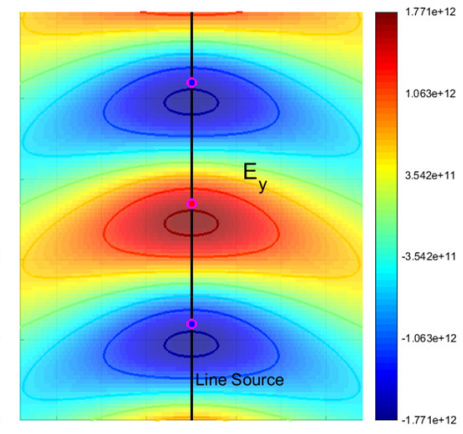

(b) $h=100$ mils
Figure 4: $\left|\vec{E}_{y}\right|$ magnitude plot, top view

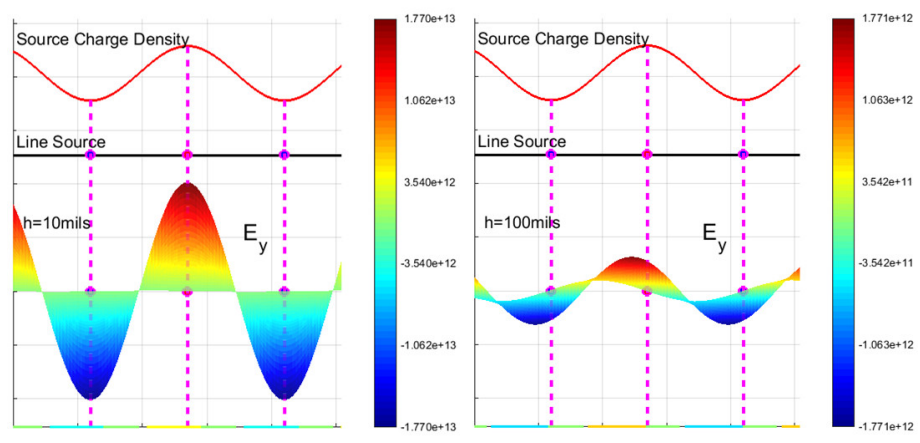

(a) $h=10 \mathrm{mils}$

(b) $h=100$ mils

Figure 5: $\left|\vec{E}_{y}\right|$ magnitude plot, side view

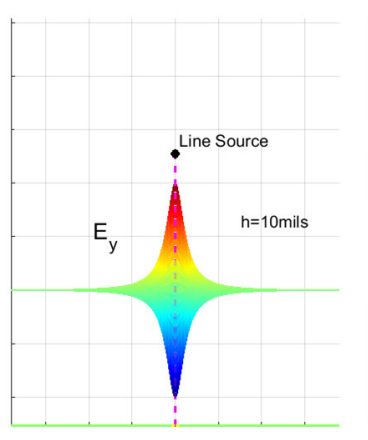

(a) $h=10 \mathrm{mils}$

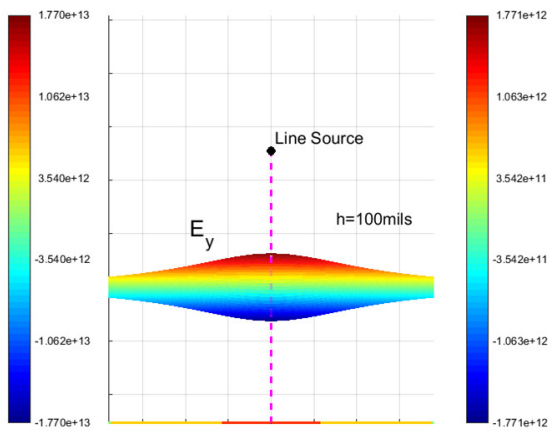

(b) $h=100$ mils
Figure 6: $\left|\vec{E}_{y}\right|$ magnitude plot, front view 
In addition to showing that the magnitude of field intensity at $\vec{x}$ is inversely proportional to its distance from $\vec{x}^{\prime}$, these electric field intensity plots, Figure 3 to Figure 6 , show the time retardation due to finite propagation velocity of fields. It is apparent that the peak of field intensity lags behind the peak of the source, and the delay is proportional to the distance, resulting in a "new moon" field pattern on the observation plane. This is more obvious with larger $h$ as the field has been spread out further when it arrives at the reference plane such as in Figures 3(b) and 4(b).

For the same study case, we can observe the field magnitude on the cross section of the model, as indicated in Figure 7. In this case, we set $h=100$ mils and set source frequency to 100 $\mathrm{GHz}$ and $10 \mathrm{GHz}$, respectively in Figure 8 and Figure 9.

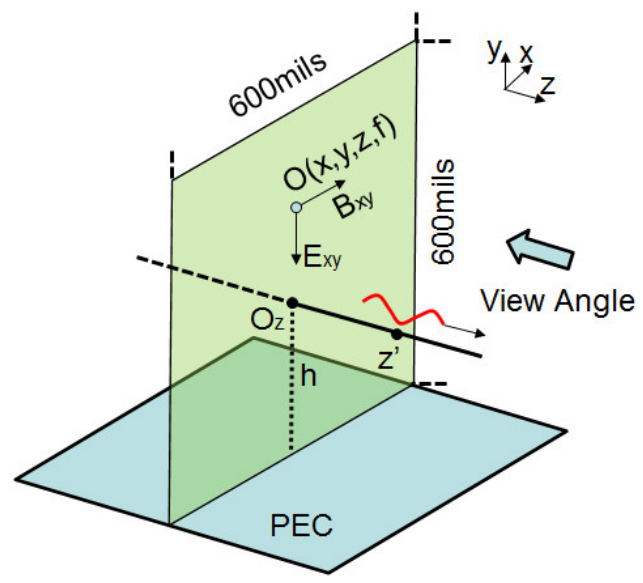

Figure 7: Case Study

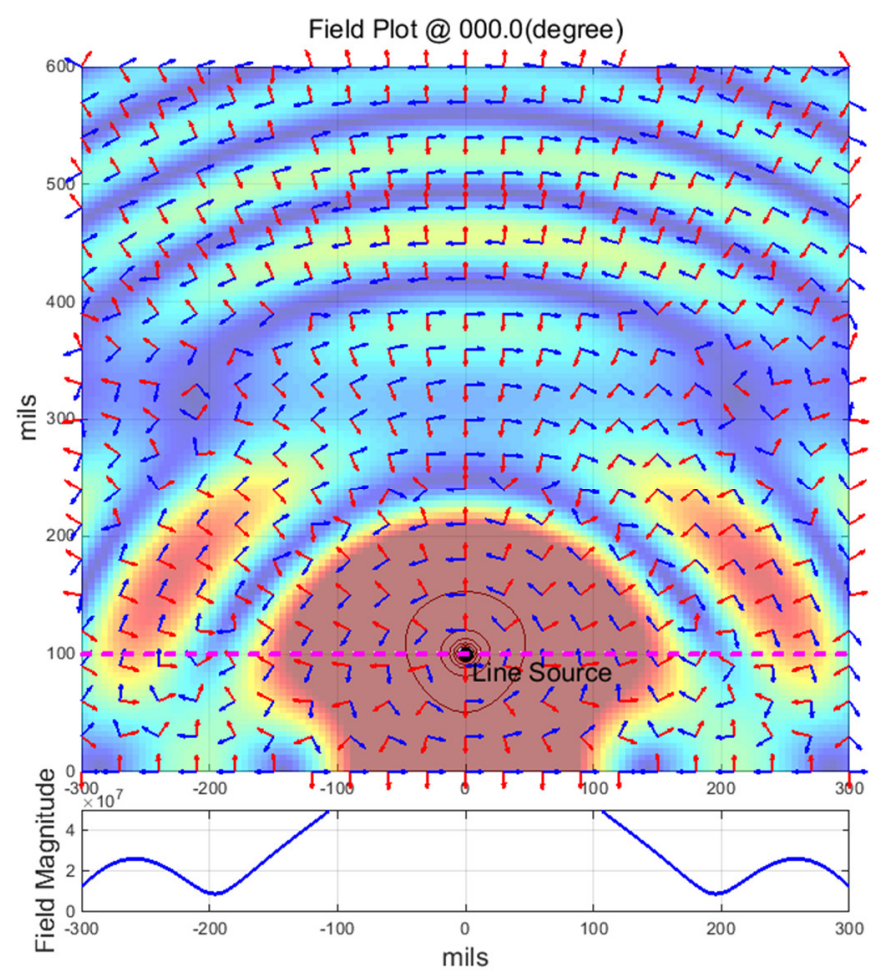

Figure 8: Field plot for $100 \mathrm{GHz}$

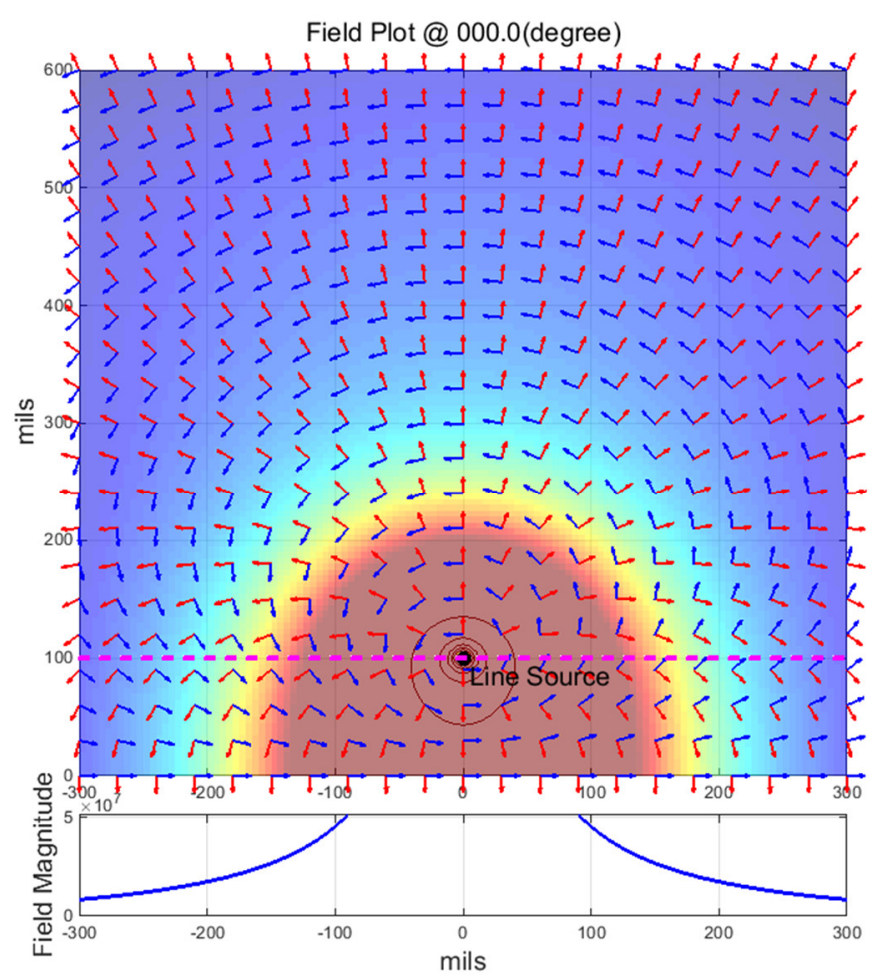

Figure 9: Field plot for $10 \mathrm{GHz}$

In these field plots, the background color represents the magnitude of the field where peak magnitude is red and minimum magnitude is blue. The red arrows indicate the direction of the $\vec{E}_{x y}$ field (electrical field on the $\mathrm{x}-\mathrm{y}$ observation plane) and the blue arrows indicate the direction of the $\vec{B}_{x y}$ field (magnetic field on the x-y observation plane).

The direction of $\vec{E}_{x y}$ (red arrows) and $\vec{B}_{x y}$ (blue arrows) in both figures shows that the energy is propagating in the same direction for any point on the observation plane; however, the direction of fields are not uniform for $100 \mathrm{GHz}$. This is due to time retardation of the fields. The total field is a sum of the original fields directly from the line source and the reflected field from the PEC reference plane. The total field at a given observation location is dominated by a certain section of the line source due to time retardation and the dominating section is related to the distance of the observation location to line source. If the source is varying at a very high frequency, there is more variation in the dominating sections on the line source for the observation area, some of which segments may even be on different parity. This creates an irregular field pattern and opposite direction of the fields for $100 \mathrm{GHz}$ source as shown in Figure 8. Conversely shown in Figure 9, if the source is varying slower there is minor variation in the dominating sections on the line source for the observation area; therefore, the total field is more uniform. Note that the direction of electric field intensity at the bottom of both Figure 8 and Figure 9 is perpendicular to the PEC as required.

Electromagnetic fields near the transmission line are the key to understanding the performance of the transmission line. As with field patterns in Figure 8 and Figure 9, there are unique 
characteristics for transmission line at high frequency. This can be studied analytically by using the retarded solution of the fields introduced in the previous section of this paper and by using the simulation program developed by the authors.

\section{Simulation Case Study}

In the previous section, it was demonstrated how to solve the electromagnetic fields near a transmission line using the retarded solution. In this section, these field solutions will be used to study the RLGC model which is a common technique for representing transmission line behavior. As in Section 2, and illustrated in Figure 7, a simplified representation of a transmission line is assumed where a lossless, thin, and uniform line source along the $z$ axis is placed above a flat, PEC plane. The medium is again assumed to be uniform and lossless with $\varepsilon=4 \varepsilon_{0}$ and $\mu=\mu_{0}$. Here, the height above the reference plane, $h$, is chosen to be 100 mils which is significant enough to invalidate the Classical Model (generally assumed to be one tenth of a wave length ${ }^{[10]}$ ).

To simulate a transmission line, the propagation constant $\gamma$ and characteristic impedance $Z_{o}$ are needed. For a uniform transmission line along the $z$ direction, the voltage and current at a given location $z$ is $V(z)=V_{+} e^{-\gamma z}+V_{-} e^{+\gamma z}$ and $I(z)=I_{+} e^{-\gamma z}+I_{-} e^{+\gamma z}$, where $V_{+} e^{-\gamma z}$ and $I_{+} e^{-\gamma z}$ are the voltage and current propagating in the $+z$ direction; $V_{-} e^{+\gamma z}$ and $I_{-} e^{+\gamma z}$ are the voltage and current propagating in the $-z$ direction. With this, the characteristic impedance is $Z_{o}=V_{+} / I_{+}=-V_{-} / I_{-}$, which is the ratio of voltage and current propagating along one direction.

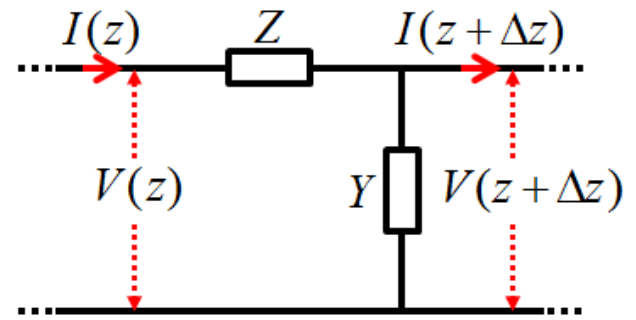

Figure 10: Impedance and Admittance network

As shown in Figure 10, the Transmission Line is divided into multiple equal segments of $\Delta z$ in length, where $\gamma$ and $Z_{o}$ are found with the impedance $Z$ and admittance $Y$ of each segment:

$\left\{\begin{array}{l}\frac{\partial V_{ \pm} e^{\mp \gamma z}}{\partial z}=-Z \cdot I_{ \pm} e^{\mp \gamma z} \\ \frac{\partial I_{ \pm} e^{\mp \gamma z}}{\partial z}=-Y \cdot V_{ \pm} e^{\mp \gamma z}\end{array} \rightarrow\left\{\begin{array}{l}V_{ \pm}=\frac{Z}{ \pm \gamma} \cdot I_{ \pm} \\ I_{ \pm}=\frac{Y}{ \pm \gamma} \cdot V_{ \pm}\end{array} \rightarrow\left\{\begin{array}{l}\gamma=\sqrt{Z \cdot Y} \\ Z_{O}=\sqrt{Z / Y}\end{array}\right.\right.\right.$ or $\left\{\begin{array}{l}Z=\gamma \cdot Z_{O} \\ Y=\gamma / Z_{O}\end{array}\right.$

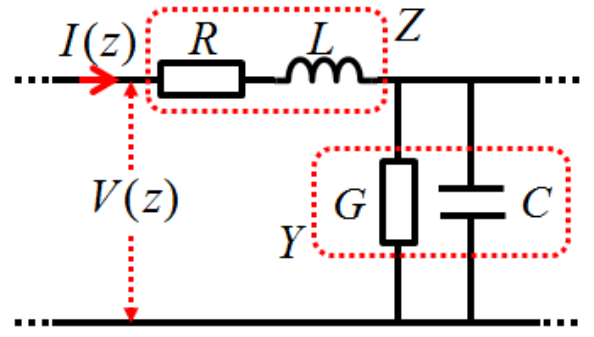

Figure 11: RLGC transmission line model segment

In a classical RLGC model, as shown in Figure 11, the impedance $Z$ and admittance $Y$ of each segment are represented by the Resistance $\mathrm{R}$, Inductance $\mathrm{L}$, Conductance $\mathrm{G}$ and Capacitance $\mathrm{C}$, where $Z=R+j \omega L, Y=G+j \omega C$ with the underlining assumption that $\mathrm{R}, \mathrm{L}, \mathrm{G}, \mathrm{C}$ parameters are positive, real values. One key assumption to establish the cascaded segments as a good representation of the transmission line is to keep each segment small enough compared to the wavelength of the frequency of interest which is application dependent. To resolve this, the classical RLGC modeling technique introduces the per unit length $\mathrm{R}$, L, G, C parameters, $R_{p u l}, L_{p u l}, G_{p u l}, C_{p u l}$, and multiplies the length of segment $\Delta z$ respectively to them to obtain $\mathrm{R}, \mathrm{L}, \mathrm{G}$, $\mathrm{C}$ values for an application, that is $R=\Delta z \cdot R_{p u l}, L=\Delta z \cdot L_{p u l}$, $G=\Delta z \cdot G_{\text {pul }}, C=\Delta z \cdot C_{\text {pul }}$. In summary, for classical RLGC model:

$$
\begin{array}{ll}
R \text { parameter }=\operatorname{real}(Z)=\Delta z \cdot R_{p u l} & Z=R+j \omega L \\
L \text { parameter }=\operatorname{imag}(Z) / \omega=\Delta z \cdot L_{p u l} \quad \text { and } & Y=G+j \omega C \\
G \text { parameter }=\operatorname{real}(\mathrm{Y})=\Delta z \cdot G_{p u l} & \gamma=\sqrt{Z \cdot Y} \\
C \text { parameter }=\operatorname{imag}(Y) / \omega=\Delta z \cdot C_{p u l} & Z_{O}=\sqrt{Z / Y}
\end{array}
$$

With the electromagnetic fields near a transmission line solved by the retarded solution, applying their physical meaning to $R_{p u l}, L_{p u l}, G_{p u l}, C_{p u l}$ leads to a different result. From a physical point of view, $R_{p u l}$ is often referred to as the electrical resistance due to the transmission line material's conductivity, and $G_{p u l}$ is referred to as the electrical loss due to surrounding material. In a simplified model with assumptions of lossless transmission line as well as lossless surrounding material, resistance and conductance can be assigned as $R_{p u l}=0$ and $G_{p u l}=0$. According to their definitions $^{[7]}, L_{p u l}$ is the ratio of the total magnetic flux surrounding a unit segment (perpendicular to the segment and extending to $\infty$ ) to the current on such segment, and $C_{p u l}$ is the ratio of the charge on a unit segment over the voltage of such segment. Since the total magnetic flux and the voltage are integral products of the fields transmission line and such fields are solved by the retarded solution as demonstrated in Section 2, the $L_{p u l}$ and $C_{p u l}$ for the simplified transmission line model setup in Section 2 are:

$$
\begin{aligned}
& L_{p u l}=\frac{\mu}{4 \pi} \int_{a}^{\infty} \int_{L_{1}}^{L_{2}}\left(x-x^{\prime}\right) e^{-j k z^{\prime}}\left[\left(\frac{e^{-j k R}}{R^{3}}-\frac{e^{-j k R_{i m g}}}{R_{i m g}^{3}}\right)+j k\left(\frac{e^{-j k R}}{R^{2}}-\frac{e^{-j k R_{i m g}}}{R_{i m g}^{2}}\right)\right] d z^{\prime} d x \\
& C_{p u l}=\frac{4 \pi \varepsilon}{\int_{a}^{\infty} \int_{L_{1}}^{L_{2}}\left(x-x^{\prime}\right) e^{-j k z^{\prime}}\left[\left(\frac{e^{-j k R}}{R^{3}}-\frac{e^{-j k R_{i m g}}}{R_{i m g}^{3}}\right)+j k\left(\frac{e^{-j k R}}{R^{2}}-\frac{e^{-j k R_{i m g}}}{R_{i m g}^{2}}\right)\right] d z^{\prime} d x}
\end{aligned}
$$


where $a$ is the radius of the transmission line, $L_{1}$ and $L_{2}$ are the starting and ending location of the transmission line, and $k=\omega / v$. Additionally, we define $R$ (not to be confused with the resistance parameter, R) as the distance from the observation point to an integral segment, and $R_{i m g}$ as the distance from the observation point to the mirror image of the same segment with respect to the reference plane. This image line source is used to assure that fields on the PEC are orthogonal to that reference plane.

For $L_{p u l}$ and $C_{p u l}$ there is a common term in the solution: $\int_{a}^{\infty} \int_{L_{1}}^{L_{2}}\left(x-x^{\prime}\right) e^{-j k z^{\prime}}\left[\left(\frac{e^{-j k R}}{R^{3}}-\frac{e^{-j k R_{i m g}}}{R_{i m g}^{3}}\right)+j k\left(\frac{e^{-j k R}}{R^{2}}-\frac{e^{-j k R_{i m g}}}{R_{i m g}^{2}}\right)\right] d z^{\prime} d x$

It has been shown ${ }^{[1,3]}$ that this term becomes more important as a function of $\omega R / v$ according to the table below:

Table 1: Values of $\omega R / v$ in typical PCB environment

\begin{tabular}{|c|c|c|c|}
\hline & $f\left(H_{z}\right)$ & $\begin{array}{l}\omega R / v \text { with } \\
\boldsymbol{R}=\mathbf{2 0} \text { mils }\end{array}$ & $\begin{array}{l}\omega R / v \text { with } \\
\boldsymbol{R}=5 \text { mils }\end{array}$ \\
\hline$D C$ & 0 & 0.000 & 0.000 \\
\hline $10 \mathrm{MHz}$ & $1.0 \times 10^{8}$ & 0.002 & 0.001 \\
\hline $1 \mathrm{GHz}$ & $1.0 \times 10^{9}$ & 0.021 & 0.005 \\
\hline $10 \mathrm{GHz}$ & $1.0 \times 10^{10}$ & 0.213 & 0.053 \\
\hline $20 \mathrm{GHz}$ & $2.0 \times 10^{10}$ & 0.426 & 0.106 \\
\hline $100 \mathrm{GHz}$ & $1.0 \times 10^{11}$ & 2.129 & 0.532 \\
\hline
\end{tabular}

Thus, for low frequencies and small values of $R$ (e.g. 5 mils), we may use the approximation $k=\omega / v=0$ (the static case) and resort to the classic RLGC model. However, at high frequencies (i.e. large values of $\omega R / v$ ) the authors define this common term as $|P| e^{j \phi(\omega)}$, where $|P|$ is the magnitude and $\phi(\omega)$ is the phase shift that accounts for retardation in the time domain. Furthermore, we can see from the four equations below that the classic RLGC model must be modified to account for time retardation effects as $\phi(\omega)$ becomes significantly greater than zero.

It can also be shown that $\phi(\omega)$ increases as $k=\omega / v$ increases. With this common term, we can see that $L_{p u l}$ and $C_{p u l}$ are also complex if the source is not static and we can write: $L_{p u l}=$ $\left|L_{\text {pul }}\right| e^{j \phi(\omega)}$ and $C_{p u l}=\left|C_{\text {pul }}\right| e^{-j \phi(\omega)}$, where: $\left|L_{\text {pul }}\right|=\frac{\mu}{4 \pi}|P|$ and $\left|C_{p u l}\right|=\frac{4 \pi \varepsilon}{|P|}$. With $Z=R+j \omega L, Y=G+j \omega C$ and $R=\Delta z \cdot R_{p u l}$, $L=\Delta z \cdot L_{p u l}, G=\Delta z \cdot G_{p u l}, C=\Delta z \cdot C_{p u l}$, we have:

$R$ parameter $=\operatorname{real}(Z)=\Delta z * \beta$ pul $-\omega \mid \Delta z *$ bul $\mid \sin \phi(\omega)$

$L$ parameter $=\operatorname{imag}(Z) / \omega=\mid \Delta z *$ bul $\mid \cos \phi(\omega)$

G parameter $=\operatorname{real}(Y)=\Delta z *$ frul $+\omega\left|\Delta z *{ }_{p} G_{\text {qul }}\right| \sin \phi(\omega)$

$C$ parameter $=\operatorname{imag}(Z) / \omega=\mid \Delta z *$ ful $\mid \cos \phi(\omega)$

The authors denote the model using these RLGC parameters as the RLGC(p) model so to distinguish from the classical RLGC model as described in the beginning of this section. For a more detailed discussion, the authors refer the reader to section 4.2 of reference [1].

A key characteristic of the RLGC(p) model is that it carries extra terms in $\mathrm{R}$ and $\mathrm{G}$ parameters from the imaginary part of inductance and capacitance. The extra terms $-\omega \mid \Delta \mathrm{z}$. $L_{p u l} \mid \sin \phi(\omega)$ and $\omega\left|\Delta \mathrm{z} \cdot C_{p u l}\right| \sin \phi(\omega)$ are in opposite polarity so one appears as an energy source and the other appears as an energy consumer. The energy "produced" by one is exactly the same as the energy "consumed" by the other so that the unit is still energy neutral, and thus energy is conserved. Due to time retardation and according to the definition of inductance and capacitance, part of the energy generated by preceding segments is accounted for in the present segment which appears as an energy "producer" for the present segment. Additionally, part of the energy generated by the present segment is accounted for by the next segment which appears as an energy "consumer" for the present segment. Through addressing the time retardation, the RLGC(p) model is able to describe this energy "produced-consumed" phenomenon. On the other hand, the classical RLGC model neglects this phenomenon.

The difference between the RLGC(p) model and the classical RLGC model may be negligible if the energy involved in "produced-consumed" phenomenon is small compared to the energy loss in the transmission line. For example in a high loss transmission line at low frequency, $R_{p u l}$ and $G_{p u l}$ are expected to be large and dominate the R parameter and $G$ parameter as defined in the RLGC(p) model, thus the classical RLGC model may differ by only a small percentage. On the contrary for a low loss transmission line at high frequency, the two models diverge as the $\mathrm{R}$ parameter and $\mathrm{G}$ parameter in $\operatorname{RLGC(p)~can~be~non-zero~and~dominated~by~the~extra~}$ terms introduced as the imaginary part of $L_{p u l}$ and $C_{p u l}$. For one such transmission line as used in Section 2, the $\mathrm{R}$ parameter actually becomes negative. The classical RLGC model cannot handle negative parameters and this causes errors compared to a FEM solution produced by a commercial 3D FEM field solver. The RLGC(p) model is well aligned with the FEM solution as shown in Figures $12-19$.

To further demonstrate the difference between the RLGC(p) model and the classical RLGC model, we take the simplified transmission line as described in Section 2. In this comparison, we plot the $\mathrm{S}$ parameter of a $500 \mathrm{mil}$ transmission line section between the frequencies $88 \mathrm{GHz}$ and $95 \mathrm{GHz}$ obtained by the following methods:

Case $<0>$ : the $S$ parameter solution from a commercial 3D FEM field solver (ANSYS HFSS). We consider this the reference for other cases.

Case $<1>$ : the $\mathrm{S}$ parameter generated by the RLGC(p) model extracted from field data of the authors' MATLAB implementation of the Retarded Solution.

Case $<2>$ : the $\mathrm{S}$ parameters generated by the classical RLGC model extracted from a 2D field solver's (ANSYS Q2D) field solution (Time Retardation ignored).

Case $<3>$ : the $\mathrm{S}$ parameters generated by the classical RLGC model extracted from field solution of Case $<0>$.

Case $<4>$ : the $\mathrm{S}$ parameters generated by the RLGC(p) model extracted from field solution of Case $<0>$.

Although a numerical method is often more computationally intensive than an analytical method, the numerical method has other advantages such as solving complex geometries; 
we focus mainly on comparing the accuracy of the results produced by several methods with each method configured to serve that purpose. It is beyond the scope of this paper to compare other aspects of each method such as computational efficiency and resources.

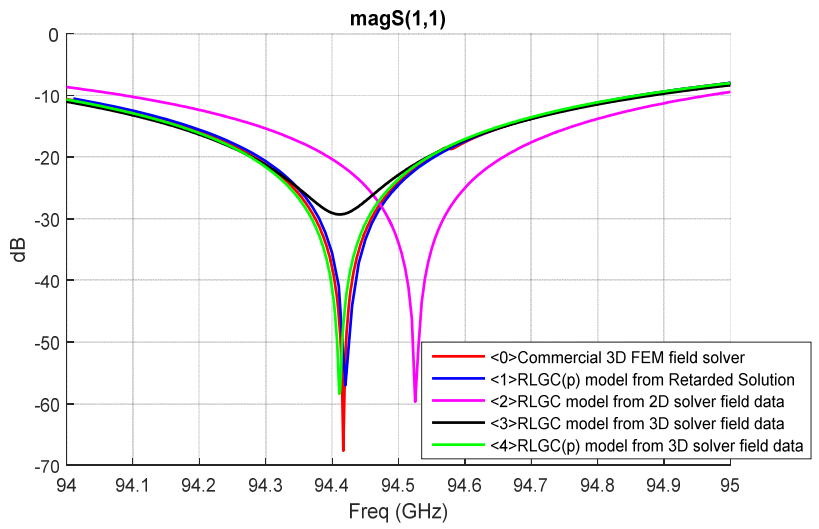

Figure 12: Magnitude of S11 from $94 \mathrm{GHz}$ to $95 \mathrm{GHz}$

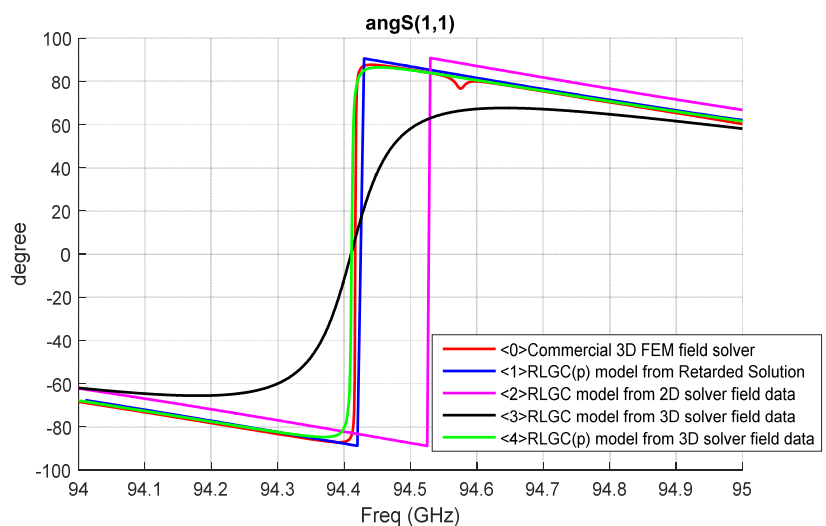

Figure 13: Phase of S11 from $94 \mathrm{GHz}$ to $95 \mathrm{GHz}$

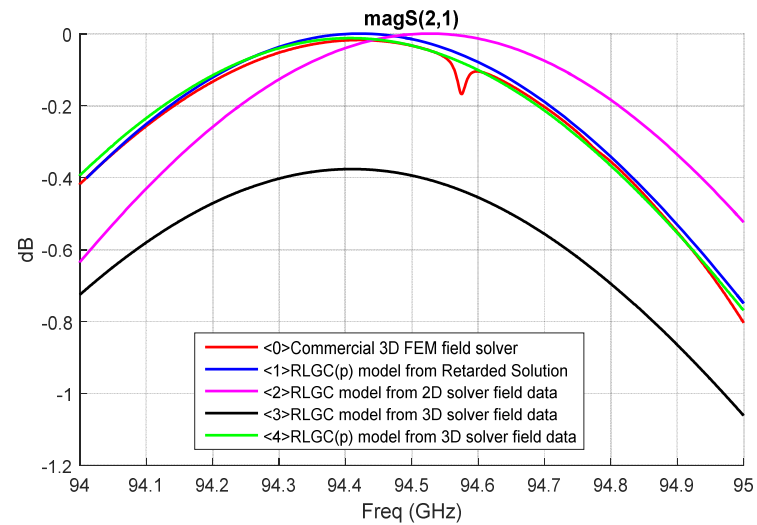

Figure 14: Magnitude of S21 from $94 \mathrm{GHz}$ to $95 \mathrm{GHz}$

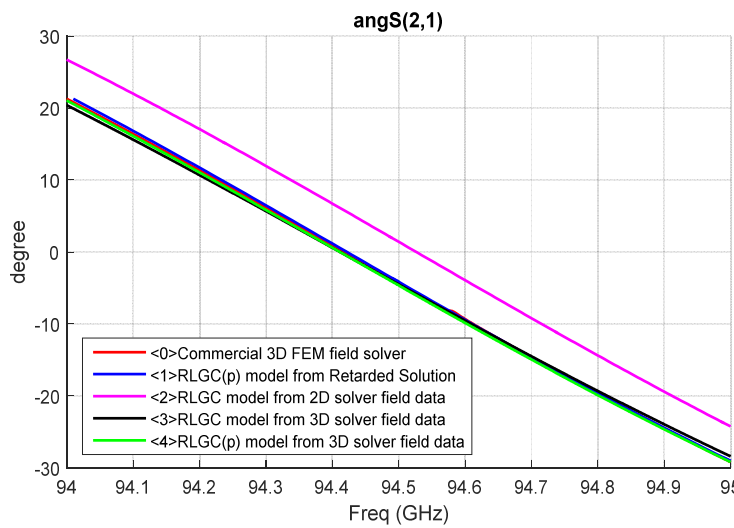

Figure 15: Phase of S21 from $94 \mathrm{GHz}$ to $95 \mathrm{GHz}$

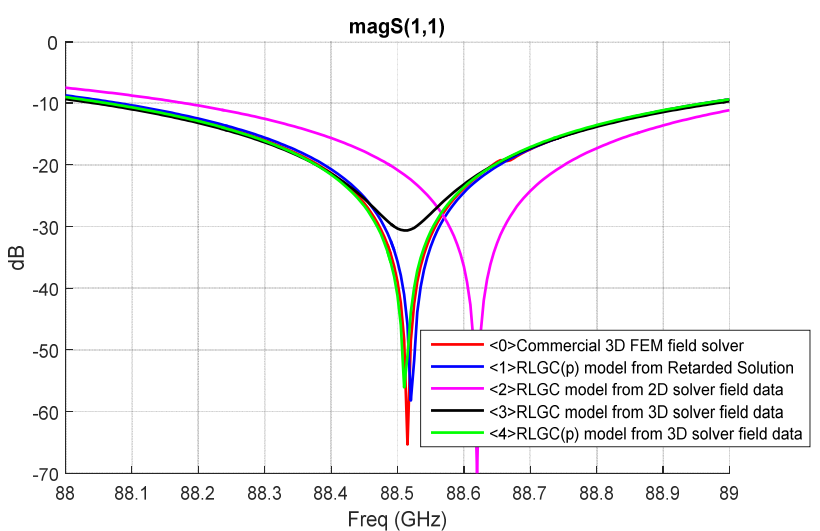

Figure 16: Magnitude of S11 from $88 \mathrm{GHz}$ to $89 \mathrm{GHz}$

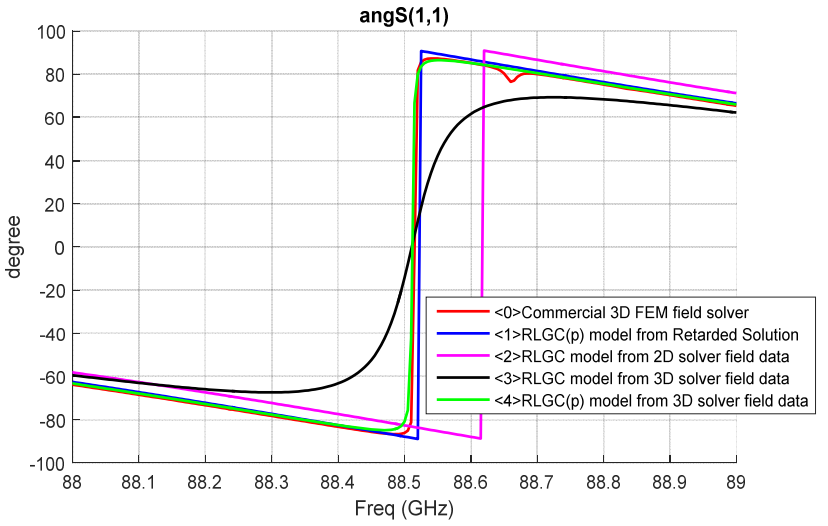

Figure 17: Phase of S11 from $88 \mathrm{GHz}$ to $89 \mathrm{GHz}$

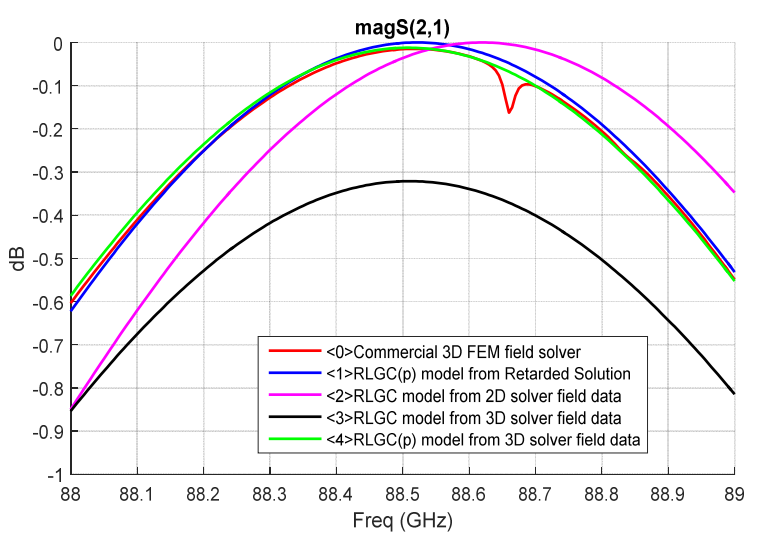

Figure 18: Magnitude of S12 from $88 \mathrm{GHz}$ to $89 \mathrm{GHz}$ 


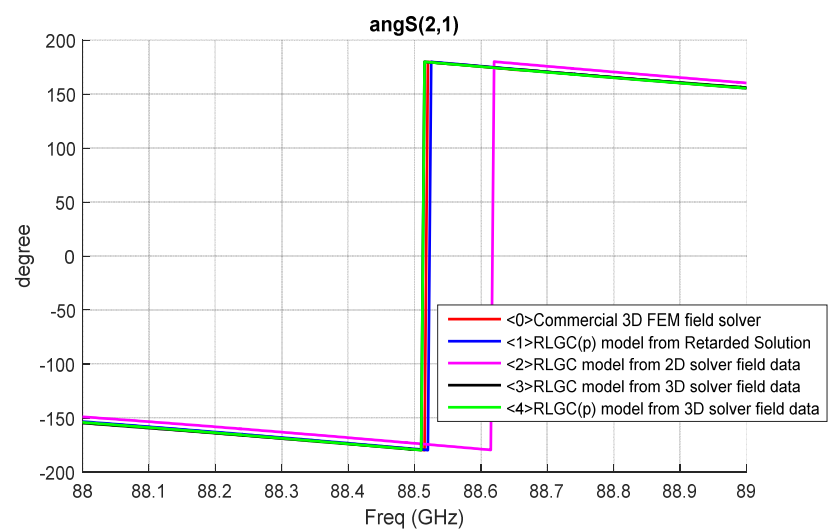

Figure 19: Phase of S12 from $88 \mathrm{GHz}$ to $89 \mathrm{GHz}$

\section{Discussion}

From Figure 12 to Figure 19, it is shown that an RLGC(p) model is effective in representing the transmission line, its performance is in good agreement to the $S$ parameters of a commercial 3D FEM field solver (Case $<0>$, Case $<1>$ and Case $<4>$ ). Comparing Case $<3>$ and Case $<4>$ shows the differences between a classical RLGC model and a RLGC(p) model; in that, the classical RLGC model trends well but is not as accurate when compared to the RLGC(p) model. It should be emphasized that Case $<1>$ and Case $<3>$ are generated from the same field data of the 3D FEM field solver used in Case $<0>$. In Case $<2>$, the classical RLGC model from 2D field solver is incorrect for the propagation constant and characteristic impedance because time retardation was not taken into account. This tells us that ignoring time retardation at high frequency results in error.

Regarding the magnitude of S12 in Figure 14 and Figure 18, the insertion loss of the transmission line, Case $<3>$ is drastically different from Case $<0>$ because the $3 \mathrm{D}$ solver "corrects" the negative parameters, in this case the $\mathrm{R}$ parameter, for the classical RLGC model with the intention of preserving passivity. However, this "correction" disturbs the energy state as it eliminates the energy source, a negative $R$ parameter, but preserves the energy consumer in $G$ parameter. We cannot preserve the energy state by eliminating both the energy source and consumer in R and $G$ parameter because it is effectively ignoring time retardation. As shown in Case $<2>$, this creates an incorrect propagation constant and characteristic impedance. The best approach is to make no "correction" as done in RLGC(p) model. As explained in Section 3, time retardation will create a complementary energy source and energy consumer, the passivity enforcement should avoid correcting such an energy source.

\section{Conclusions}

As demonstrated, the time retardation of electric and magnetic field propagation results in complex capacitance and inductance. The imaginary part of capacitance and inductance affect the RLGC parameters and cause the classical RLGC parameters at high frequencies to be inconsistent with their DC counterparts. A new RLGC(p) model is proposed to handle this inconsistency. It is necessary to use RLGC(p) model instead of the classical RLGC model to simulate a transmission line where retardation is significant. In this paper's transmission line example, the Field Retardation is considered significant, and the RLGC(p) model is shown to be in better agreement than the classical RLGC model to an FEM solution. Future work is ongoing to extend this model to multiple conductors; as well as, explore the limitations for extremely high frequencies.

\section{References}

[1] Peng Ye, Paul G. Huray, "Applying the Retarded Solution of Electromagnetic Fields to PCB Transmission Line RLGC Modeling", Ph.D. Dissertation, University of South Carolina, 2015

[2] John David Jackson, "Classical Electrodynamics", Section 7.11, Wiley, $3^{\text {rd }}$ edition, 1998.

[3] Oleg D. Jefimenko, "Causality Electromagnetic Induction and Gravitation", Electret Scientific Co; $2^{\text {nd }}$ edition, 2000.

[4] Paul G. Huray, "The Foundations of Signal Integrity", pg. 33, Wiley-IEEE Press, $1^{\text {st }}$ edition, 2010.

[5] Paul G. Huray, "Maxwell's Equations", Wiley-IEEE Press, $1^{\text {st }}$ edition, 2010.

[6] Richard P. Feynman, Robert B. Leighton, Matthew Sands, "The Feynman Lectures on Physics, Volume 2, Section 21", Addison-Wesley, 1977.

[7] Rosa, E.B. "The Self and Mutual Inductances of Linear Conductors", Bulletin of the Bureau of Standards 4 (2): 301-344.

[8] Albert Ruehli, "Partial Element Equivalent Circuit (PEEC) Method and Its Application in the Frequency and Time Domain", in Proc. Electromagn. Compat. Symp., Aug. 19-23, 1996, pp. 128-133.

[9] Madhusudanan K. Sampath, "On Addressing the Practical Issues in the Extraction of RLGC Parameters for Lossy Multi Conductor Transmission Lines using Sparameter Models", Proceedings of the 16th Topical Meeting on the Electrical Performance of Electronic Packaging, pp. 259-262 (Oct. 2008).

[10] Sofiane Chabane, Philippe Besnier, Marco Klingler, “ $A$ Modified Enhanced Transmission Line Theory Applied to Multi Conductor Transmission Lines", IEEE Transactions on Electromagnetic Compatibility, Early access, 2016 